\title{
Involvement of neurokinin 1 receptor within the cerebrospinal fluid-contacting nucleus in visceral pain
}

\author{
CHAO ZHANG, YING LI, XIN WANG, YAN FEI and LICAI ZHANG \\ Jiangsu Province Key Laboratory of Anesthesiology, Xuzhou Medical College, Xuzhou, Jiangsu 221002, P.R. China
}

Received March 2, 2016; Accepted February 13, 2017

DOI: $10.3892 / \mathrm{mmr} .2017 .6499$

\begin{abstract}
Studies have shown that the cerebrospinal fluid-contacting nucleus (CSF-CN) may be associated with the transduction and regulation of pain signals. However, the role of the CSF-CN remains to be elucidated. Emerging evidence has suggested that neurokinin 1 receptor (NK1R) is important in the development of visceral pain and hyperalgesia, however, whether NK1R exists in the CSF-CN and its exact role in visceral pain remain to be fully elucidated. In the present study, double-labeled immunofluorescence staining and western blot analysis were performed to investigate this. It was revealed that NK1R was distributed in the CSF-CN Following the induction of visceral pain by formalin instillation, NK1R in the CSF-CN was upregulated. In addition, by observing the behaviors of rats subjected to visceral pain, it was found that visceral pain was relieved by lateral intracerbroventricular injection of the NK1R antagonist, RP67580. These data provided a broader understanding of the role of NK1R in the CSF-CN and demonstrated that the CSF-CN was involved in acute visceral pain via the regulation of NK1R.
\end{abstract}

\section{Introduction}

Visceral pain refers to a type of pain, which occurs following noxious stimulation surrounding internal organs. Accompanied by ambiguous location and internal organ cramps, it causes severe pain and causes muscle pain in referred areas, which affects the quality of life of patients and substantially increases medical treatment burden. At present, how visceral pain occurs and the pathway of visceral noxious information transmission remain to be fully elucidated due to limitations in experimental techniques (1-4).

Correspondence to: Professor Licai Zhang, Jiangsu Province Key Laboratory of Anesthesiology, Xuzhou Medical College, 209 Tongshan Road, Xuzhou, Jiangsu 221002, P.R. China

E-mail: licaizhang01@163.com

Key words: cerebrospinal fluid-contacting nucleus, neurokinin 1 receptor, visceral pain, RP67580, immunohistochemistry
Substance P (SP), first identified in 1931, is a type of neurokinin. It is well documented that SP is an excitatory neurotransmitter released by the first stage of peripheral nociceptive afferent fibers and is involved in pain transmission. As its specific receptor, neurokinin 1 receptor (NK1R) has been demonstrated to be important in the development of pain and hyperalgesia. However, how NK1R functions when visceral pain occurs remains to be elucidated $(5,6)$. The cerebrospinal fluid (CSF)-contacting nucleus (CSF-CN), the neurons of which contact the internal CSF, was first identified and named at the Jiangsu Province Key Laboratory of Anesthesiology, Xuzhou Medical College (Xuzhou, China). Using cholera toxin B subunit conjugated to peroxidase (CB-HRP), CSF-CN was successfully labeled and distributed in the ventral periaqueductal central gray matter of the brainstem $(7,8)$. The CSF-CN is unique owing to its specific structure. The cell bodies of these neurons are in the brain parenchyma, whereas their projections are in contact with the CSF $(9,10)$. Previous studies have demonstrated that there are subtle changes in the chemical composition of CSF when pain occurs $(11,12)$. Considering the specific structure of the CSF-CN, the present study hypothesized that the CSF and the CSF-CN have certain indivisible connections. Evidence from previous studies has supported this hypothesis, and suggested that the CSF-CN is involved in the transduction and regulation of pain signals $(13,14)$. However, the role of CSF-CN remains to be fully elucidated. The present study aimed to investigate the possible functions of NK1R within the CSF-CN under conditions of visceral pain in order to provide a feasible solution for the clinical treatment of visceral pain.

\section{Materials and methods}

Animals. All experiments were performed according to the regulations of The Committee for the Ethical Use of Laboratory Animal of Xuzhou Medical College. Male Sprague-Dawley rats provided by the Experimental Animal Center of Xuzhou Medical College (license number, SYXK 2002-0038) were 8-9 weeks old and weighed 250-300 g. The rats were randomly assigned into three groups, each containing six animals. The rats in the visceral pain group were subjected to lower colon instillation of 5\% formalin; in the sham group, rats received instillation of saline; in the RP67580 group, a specific NK1R antagonist was injected into the lateral ventricle (LV) of rats $24 \mathrm{~h}$ prior to visceral pain induction. All animals were 
placed in separate rooms, in which conditions and light were controlled $\left(23 \pm 1^{\circ} \mathrm{C} ; 12 / 12 \mathrm{~h}\right.$ dark/light cycle with light on at 8:00 a.m.), and food and water were available ad libitum.

Formalin instillation. In the animal experiments, visceral pain was induced by rectal infusion with dilute formalin. In order to allow the rats to regain consciousness as soon as possible, a small quantity of halothane (induction at 3\%, then $1.5 \%$ in a mixture of $2: 3$ nitrous oxide and 1:3 oxygen) was used. While under anesthesia, the animal was suspended by its tail ( $<5 \mathrm{~min})$. Subsequently, a polyethylene tube, which was wrapped in surgical tape at $\sim 30 \mathrm{~mm}$ from the edge, was inserted through the anus. A 5\% formalin solution or saline $(100 \mu \mathrm{l})$ was injected through the tube slowly to prevent leakage (15).

Visceral pain behavior score assessment. All animals were placed in separate observation boxes for adaptation $30 \mathrm{~min}$ prior to the behavioral assessments. On regaining consciousness, the rats were observed for $3 \mathrm{~h}$ to record pain-associated behaviors. The visceral pain-associated behaviors included licking of the abdomen (A), back stretching (B), contraction of flanks (C), and contraction of whole body (D). Based on the duration of the contraction, the contraction of the whole body was divided into three levels of $<30 \mathrm{sec}$ (W1), 30-60 sec (W2) and $>1 \mathrm{~min}$ (W3). Using the following formula, the visceral pain score (PS) was calculated: $\mathrm{PS}=\mathrm{L}+2 \mathrm{~B}+3 \mathrm{C}+4 \mathrm{~W} 1+5 \mathrm{~W} 2+6 \mathrm{~W} 3$ (16).

Nociceptive assessment. The animals were placed in grid-bottomed cages for $30 \mathrm{~min}$ for adaption prior to the evaluation of allodynia. Subsequently, to determine the hyperalgesia of the referred area, von Frey hairs (VFHs; Semmes-Weinstein Monofilaments, North Coast Medical, Inc. San Jose, CA, USA) were used. On stimulation of the abdomen with increasing force $(0.16,0.4,0.6,1,1.4,2,4,6,8,10$ and $15 \mathrm{~g})$, the rats showed withdrawal responses, which included sharp abdominal retraction, licking or scratching the site of application of the hair and jumping with all four paws off the floor (17). Each VFH test lasted for 6 sec, or until a withdrawal response was observed. Once a withdrawal reaction occurred, the next descending force of VFH was applied to retest the area where the former VFH was placed until no response was detected. The minimum force was recorded as the abdomen withdrawal threshold in grams. If the result of allodynia was $\leq 2 \mathrm{~g} \mathrm{VFH}$, it was considered innocuous to the rats.

Drugs administration. The animals were anesthetized with sodium pentobarbital (40 mg/kg, i.p.). When immobilized in stereotaxic instrument, the rat was injected with $3 \mu 1$ of 30\% CB-HRP (Sigma-Aldrich; Merck KGaA, Darmstadt, Germany) into the $\mathrm{LV}$ according to stereotaxic coordinates (Brega, $-1.2 \pm 0.4 \mathrm{~mm}$; depth, $3.2 \pm 0.4 \mathrm{~mm}$; right to median sagittal plane, $1.4 \pm 0.2 \mathrm{~mm}) 48 \mathrm{~h}$ prior to the behavioral assessments. The NK1R antagonist, RP67580 (Tocris Biosciences, Bristol, United Kingdom) was dissolved in dimethyl sulfoxide, and 100 pmol was injected into the LV of rats in the RP67580 group $24 \mathrm{~h}$ prior to the behavioral assessments.

Immunohistochemistry and imaging. Following the intracerbroventricular injections, the rats were administered with the formalin instillation and visceral pain behavioral assessments were performed. Subsequently, the rats were deeply anesthetized by injection of pentobarbital sodium $(50 \mathrm{mg} / \mathrm{kg}$, i.p.) and then successively perfused with $150 \mathrm{ml}$ of phosphate buffered saline (PBS; $0.01 \mathrm{M}$; pH 7.4) and 4\% paraformaldehyde. Following isolation and overnight fixation, the brainstem was immersed in $30 \%$ sucrose solution for 2 days. On a cryostat microtome (Leica CM1900; Leica Microsystems GmbH, Wetzlar, Germany), all tissues were successively cut into $35 \mu \mathrm{m}$ slices in the transverse plane. The sections were then incubated in donkey serum (1:100 dilution; cat. no. 566460; Merck KGaA) for $1 \mathrm{~h}$ at $37^{\circ} \mathrm{C}$, following which the sections were treated with goat anti-CB (1:400 dilution; cat. no. 227040; Merck KGaA) and anti-rabbit NK1R (1:100 dilution; cat. no. BA3678; Wuhan Boster Biological Technology Ltd., Wuhan, China). Following reaction with goat anti-IgG coupled to Alexa 546 (1:400 dilution; cat no. A11056; Thermo Fisher Scientific, Inc., Waltham, MA, USA) and rabbit anti-IgG coupled to Alexa 488 (1:400 dilution; cat. no. A21206; Thermo Fisher Scientific, Inc.), the slices were thoroughly rinsed three times with $0.01 \mathrm{~mol} / \mathrm{ml}$ PBS at the end of each step. The tissue sections were transferred onto slides and allowed to air dry, prior to sealing with glycerol. Finally, ultrathin sections $(75 \times 25 \times 1 \mathrm{~mm})$ were prepared and examined under a laser-scanning confocal microscope (TCS SP2; Leica Microsystems). Using Image-Pro Plus (version 6.0; Media Cybernetics, Inc., Rockville, MD, USA), images were captured, cropped and adjusted.

Western blot analysis. Following the behavior assessments, the rats were sacrificed and the CSF-CN region of rat brain was removed and stored at $-80^{\circ} \mathrm{C}$. The tissues were homogenized by an electric homogenizer for $20 \mathrm{sec}$ at room temperature. Following centrifugation at $13,523 \times \mathrm{g}$ for $15 \mathrm{~min}$ at $4^{\circ} \mathrm{C}$, supernatant collection and denaturation, the lysates were electrophoresed on $8 \%$ SDS-polyacrylamide gel. According to the analysis, the semi-dry western blot transfer method was used, and the polyvinylidene difluoride membranes were blocked with $5 \%$ skim milk for $1 \mathrm{~h}$ at room temperature. The membranes were then incubated with anti-rabbit NK1R (1:500 dilution; cat. no. BA3678; Wuhan Boster Biological Technology Ltd.) and anti-rabbit GAPDH (1:5,000 dilution; cat. no. sc-25778; Santa Cruz Biotechnology, Inc., Dallas, TX, USA) at $4^{\circ} \mathrm{C}$ overnight. The membranes were washed with TBS-Tween 3 times and incubated with secondary anti-rabbit IgG antibody (1:1,000 dilution; cat. no. A0545; Sigma-Aldrich; Merck $\mathrm{KGaA}$ ) for $2 \mathrm{~h}$ at room temperature. The densities of the bands were analyzed using ImageJ software (version 1.47; National Institutes of Health, Bethesda, MD, USA).

Statistical analysis and image analysis. Quantitative immunohistochemistry was used to assess the expression of NK1R in the CSF-CN. Image analysis software (Image-Pro Plus Version 6.0; Media Cybernetics, Inc.) was used for digital image analysis. On examination of every tissue section, details were recorded on the number, area and density of immunoreactive cells. Dual labeling of neurons with CB-HRP/NK1R enabled counting via a yellow fluorescence reaction, with areas between 40 and 1,000 $\mu \mathrm{m}^{2}$. Data are expressed as the mean \pm standard deviation and were imported into Microsoft Excel 2003 (Microsoft Corporation, Redmond, WA, USA). 
Using SPSS v.13.0 (SPSS, Inc., Chicago, IL, USA), all data were analyzed using one-way analysis of variance or Student's t-test. $\mathrm{P}<0.05$ was considered to indicate a statistically significant difference.

\section{Results}

Behavior in formalin-induced visceral pain. Prior to surgery, the PS of visceral pain behavior at baseline was determined in untreated rats, which was $0.8 \pm 0.7$. Following formalin instillation, a series of discrete behavioral episodes were observed, including licking of the upper abdomen, stretching of the whole body and arching of the back against the floor. Compared with the PS of rats in the sham group, the PS of rats in the visceral pain group presented with a typical biphasic time course. The first peak occurred at $\sim 15$ min post-instillation ( $\mathrm{PS}=87.8 \pm 5.4$ ), which was 15 times higher, compared with that in the saline instillation group $(\mathrm{P}<0.05)$. The second PS peak occurred at 60 min post-instillation ( $\mathrm{PS}=36.7 \pm 3.3$ ), which was 12 times higher, compared with that of the sham group $(\mathrm{P}<0.05)$. The PS of the RP67580 group was significantly decreased at 60 min post-formalin instillation, compared with that of the visceral pain group $(\mathrm{P}<0.05$; Fig. 1A).

Mechanical allodynia in rats following formalin instillation. In the present study, the von Frey assessment was used to measure the abdomen withdrawal threshold. The values of the abdomen withdrawal threshold in rats of the visceral pain group were significantly lower $60 \mathrm{~min}$ post-formalin instillation, compared with that of the sham group $(\mathrm{P}<0.05)$. The values were significantly upregulated in rats pretreated with RP67580 (P<0.05; Fig. 1B).

Immunohistochemical analysis of NKIR in the CSF-CN of rats following formalin instillation. The CSF-CN was first identified by injecting CB-HRP, as a fluorescent tracer, into the $\mathrm{LV}$ of the rats, which was used as a reliable method to label CSF-CN. As shown in the images in Fig. 2, the neurons labeled by CB-HRP were predominantly located around the midline of the aqueduct midbrain and ventral periaqueductal gray matter of the brainstem. In order to determine whether the CSF-CN expressed NK1R, a double-labeled immunofluorescence technique was used. The results indicated that NK1R-immunoreactive neurons (green) were distributed in the CSF-CN. The total CSF-CN sample of the visceral pain group did not differ from that of the sham group $(\mathrm{P}>0.05)$. The number of simple labeled NK1R-containing neurons counted in the visceral pain group was significantly higher, compared with that in the sham group $(\mathrm{P}<0.05)$. Compared with the sham group, then number of dual-labeled CB-HRP/NK1R neurons counted in the visceral group was significantly upregulated $(\mathrm{P}<0.05$; Fig. 2; Table I).

Western blot analysis of NKIR in the CSF-CN of rats experiencing visceral pain. Based on the above-mentioned findings, the present study investigated the role of NK1R in visceral pain. Western blot analysis was used to examine the protein expression levels of NK1R, the result of which indicated that NK1R was upregulated 60 min following formalin instillation, compared with that of the sham group $(\mathrm{P}<0.05)$.
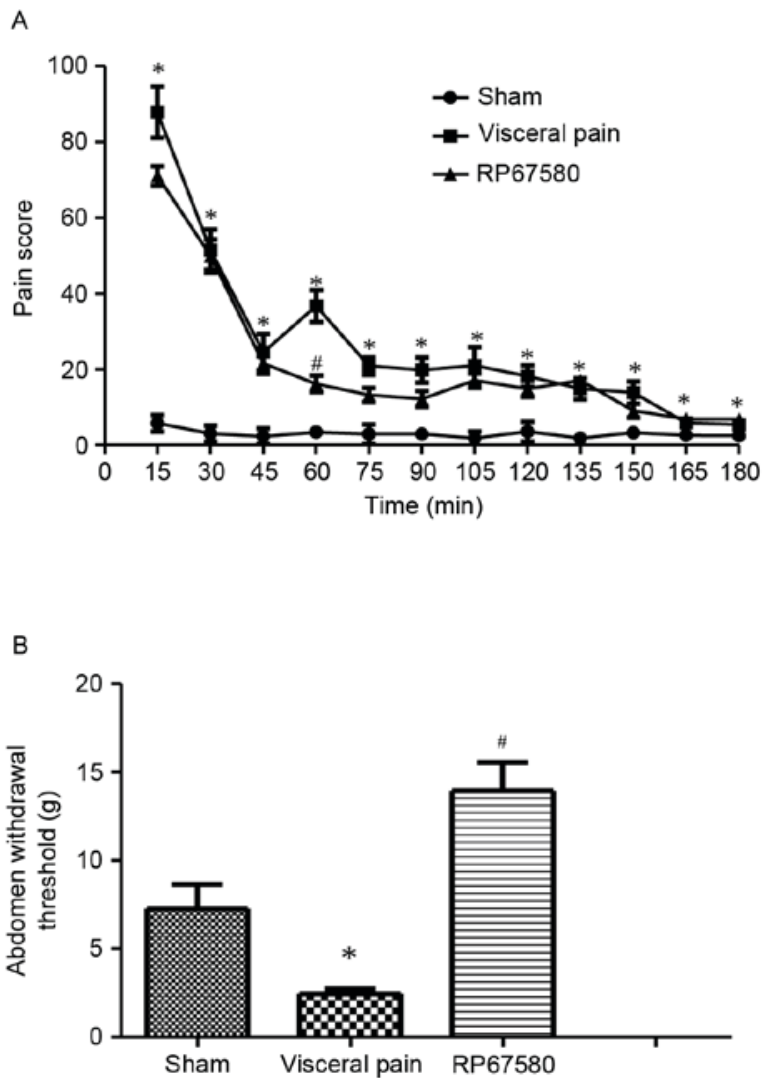

Figure 1. PS time course and abdomen withdrawal threshold assessment (A) Time course of PS in animals in response to formalin instillation or saline instillation (sham). The PS of rats in the visceral pain group was higher, compared with that of rats in thesham group at all time points. At 60 min post-formalin instillation, the PS of rats pretreated with RP67580 was decreased, compared with that of rats in visceral paingroup. (B) Abdomen withdrawal thresholds in rats at 60 min following formalin or saline instillation. The abdomen withdrawal threshold of the visceral pain group was significantly decreased, compared with that of the sham group. However, that of the RP67580 group was significantly upregulated. ${ }^{*} \mathrm{P}<0.05$ vs. sham group; ${ }^{\#} \mathrm{P}<0.05$ vs. visceral pain group. $\mathrm{PS}$, pain score.

A
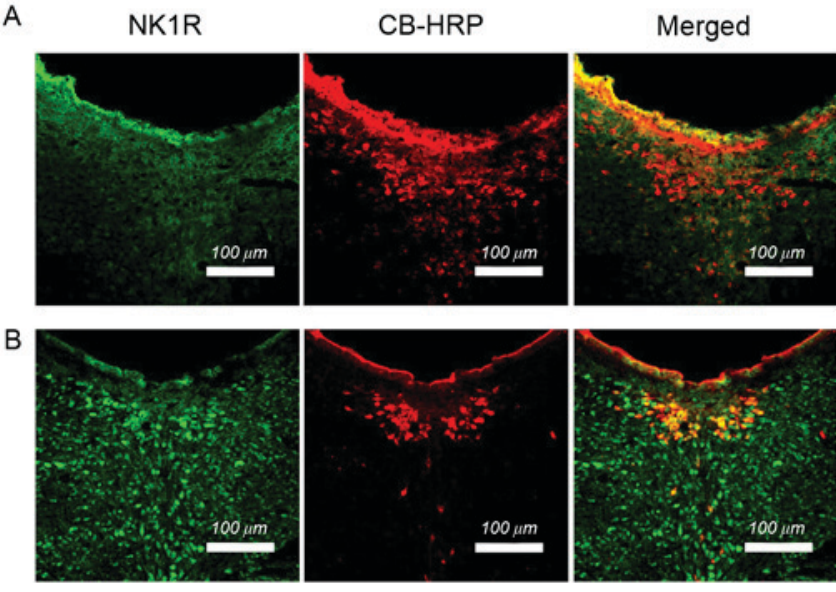

Figure 2. Dual labeling of neurons using CB-HRP/NK1R fluorescent immunohistochemistry. Images showing labeling of neurons in (A) rats in the sham group and (B) rats in the visceral pain group. NK1R, neurokinin 1 receptor; CB-HRP, cholera toxin B subunit conjugated to peroxidase.

Following LV injection of RP67580, the rats experienced significant relief from pain on formalin instillation, which 
Table I. Expression of CB-HRP, NK1R and CB-HRP/NK1R in the sham group and visceral pain group.

\begin{tabular}{lllc}
\hline Group & CB-HRP & NK1R & CB-HRP/NK1R \\
\hline Sham & $398 \pm 16$ & $114 \pm 7$ & $70 \pm 3$ \\
Visceral pain & $407 \pm 12$ & $604 \pm 12^{\mathrm{a}}$ & $353 \pm 7^{\mathrm{a}}$ \\
\hline
\end{tabular}

CB-HRP is indicative of cerebrospinal fluid-contacting nucleus. Data are presented as the mean \pm standard deviation. ${ }^{\mathrm{a}} \mathrm{P}<0.05$. NK1R, neurokinin 1 receptor; CB-HRP, cholera toxin B subunit conjugated to peroxidase.

A

$\begin{array}{lll}S \quad V P \quad R P 67580 & \\ & \text { NK1R (58 kDa) } \\ & \text { GAPDH (37 kDa) }\end{array}$

B

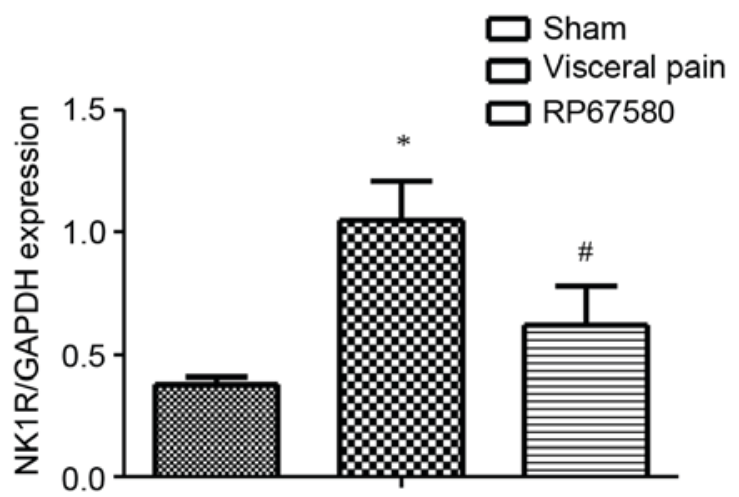

Figure 3. Protein expression of NK1R in the CSF-CN. (A) Blots showing the protein expression of NK1R in the CSF-CN. (B) Results of western blot analysis for NK1R protein in the CSF-CN. In the visceral pain group, the protein expression of NK1R in the CSF-CN was higher, compared with that of the sham group. The protein expression of NK1R in the CSF-CN of rats pretreated with RP67580 was lower, compared with that of the visceral pain group. ${ }^{~} \mathrm{P}<0.05$ vs. sham group; ${ }^{\#} \mathrm{P}<0.05$ vs. visceral pain group. NK1R, neurokinin 1 receptor; CSF-CN, cerebrospinal fluid-contacting nucleus; $\mathrm{S}$, sham; VP, visceral pain.

suggested that NK1R in the CSF-CN may be associated with the alleviation of visceral pain. Western blot analysis was performed to observe the expression of NK1R $24 \mathrm{~h}$ following the injection of RP67580. Compared with the rats in the visceral pain group, there was a significant downregulation in the expression of NK1 in the rats pretreated with RP67580 ( $\mathrm{P}<0.05$; Fig. 3).

\section{Discussion}

In previous studies, acute colitis has been used as a model to simulate visceral pain of pelvis organs, including the lower colon, rectum and bladder $(16,17)$. Through the rectal infusion of formalin, inflammatory pain can be successfully simulated in regions of regions of the pelvic organs (18). In the present study, this method was used to induce visceral pain. The results showed that the visceral pain behavior scores of rats in the visceral pain group were significantly increased at each time point, compared with those of rats in the sham group, which suggested establishment of the visceral pain model had been successful. The present study demonstrated that the intestinal perfusion of formalin mediated a biphasic reaction of visceral pain, which was similar to the reaction of formalin-induced somatic pain (19). The specific mechanism underlying this biphasic pain remains to be fully elucidated and requires further investigation.

Our previous investigations demonstrated that the CSF-CN participated in the transmission of information within the brain, with chemical messages transferred in a cell-to-cell manner or in the CSF (20). It has been found that the neurons of the CSF-CN have a specific cell structure, with cell bodies located within the brain parenchyma and projections extending into the CSF through ependymal barriers (7). In addition, signal transmission by the CSF-CN may affect the composition of the CSF, which may lead to neuromodulation or neuroendocrinal regulation (21).

NK1R is a G protein-coupled receptor, which can specifically combine with SP. It has been well documented that SP is involved in the pain regulation process (6). Several studies have suggested that CSF-CN is involved in the transmission of pain signals by the expression of SP, transient receptor potential cation channel, subfamily $\mathrm{C}$, member 6 and extracellular-signal-regulated kinase $5(10,22,23)$. In the present study, using a double-labeled immunofluorescent technique, it was observed that NK1R was expressed in the CSF-CN when visceral pain occurred, and that there were alterations in the PS and abdomen withdrawal threshold in rats exposed to formalin instillation, compared with those of naive rats. To assess the involvement of NK1R of the CSF-CN in the behavioral nociceptive symptoms due to visceral inflammation and to semi-quantitatively analyze variations in the expression ofNK1R in visceral pain, western blot analysis was used. The results showed that, at $60 \mathrm{~min}$ post-formalin instillation, when the second phase of visceral pain occurred, the protein expression level of NK1R within the CSF-CN was upregulated. In addition, RP67580, a specific NK1R antagonist, significantly alleviated visceral pain in rats and reduced the expression of NK1R, suggesting that the upregulation of NK1R may be crucial in the process of establishing inflammation-induced visceral pain. Based on these findings, it was hypothesized that NK1R was involved within the CSF-CN in visceral pain.

Previous clinical and experimental studies have confirmed the presence of NK1R on postsynaptic dorsal column (PSDC) neurons in inflammation of the colon. These findings may explain why DC lesions can relieve visceral pain in patients (16). Studies on NK1R gene-deficient mice have shown that NK1R can mediate the central nociceptive and peripheral inflammatory response. It can cause neurogenic inflammation and regulate the peripheral inflammatory responses to noxious stimuli (24). Evidence has suggested that the perception of visceral pain may be completed by an ascending excitatory pathway in the DC, particularly under 
the conditions of peripheral inflammation. There is increasing evidence that this pathway may contain an amplification loop, which enhances the responsiveness of spinal cord neurons through a descending facilitatory pathway, possibly originating in the rostroventral medulla. PSDC and other projection neurons may be affected by this amplification loop and lead to potentiation (25). AsNK1R is expressed in PSDC neurons under conditions of visceral pain, PSDC neurons may be involved in the amplification mechanism mediated by the NK1R. NK1R may regulate visceral pain through the transfer of information between the brain parenchyma and CSF, or be involved in the DC pathway via its expression in PSDC neurons. Additionally, the results of the present study showed that the visceral pain was not completely relieved following pre-injection of RP67580, which suggested that there may be other mechanisms for regulating visceral pain. A substantial number of neurons of the CSF-CN did not express NK1R, and other neurotransmitters or signaling pathways may affect the regulation of visceral pain.

In conclusion, the present study provided novel evidence that NK1R was expressed in the CSF-CN, and that NK1R within the CSF-CN may be involved in the regulation of visceral pain. However, the way in which NK1R in the CSF-CN affects the regulation of visceral pain remains to be elucidated in future investigations.

\section{Acknowledgements}

This study was supported by a grant from the National Natural Science Foundation of China (grant no. 81371243) and the Natural Science Foundation of Jiangsu Province (grant no. BK2012580).

\section{References}

1. Ness TJand Gebhart GF: Colorectal distension as a noxious visceral stimulus: Physiologic and pharmacologic characterization of pseudaffective reflexes in the rat. Brain Res 450: 153-169, 1988.

2. Ness TJ and Gebhart GF: Visceral pain: A review of experimental studies. Pain 41: 167-234, 1990.

3. Laird JM, Martinez-Caro L, Garcia-Nicas E and Cervero F: A new model of visceral pain and referred hyperalgesia in the mouse. Pain 92: 335-342, 2001.

4. Greenwood-Van Meerveld B, Gibson MS, Johnson AC, Venkova K and Sutkowski-Markmann D: NK 1 receptor-mediated mechanisms regulate colonic hypersensitivity in the guinea pig. Pharmacol Biochem Behav 74: 1005-1013, 2003.

5. Allen BJ, Rogers SD, Ghilardi JR, Menning PM, Kuskowski MA, Basbaum AI, Simone DA and Mantyh PW: Noxious cutaneous thermal stimuli induce a graded release of endogenous substance $\mathrm{P}$ in the spinal cord: Imaging peptide action in vivo. J Neurosci 17: 5921-5927, 1997

6. Steinhoff MS, von Mentzer B, Geppetti P, Pothoulakis C and Bunnett NW: Tachykinins and their receptors: Contributions to physiological control and the mechanisms of disease. Physiol Rev 94: 265-301, 2014.

7. Zhang LC, Zeng YM, Ting J, Cao JP and Wang MS: The distributions and signaling directions of the cerebrospinal fluid contacting neurons in the parenchyma of a rat brain. Brain Res 989: 1-8, 2003.
8. Liu H, Yan WW, Lu XX, Zhang XL, Wei JQ, Wang XY, Wang T, Wu T, Cao J, Shao CJ, et al: Role of the cerebrospinal fluid-contacting nucleus in the descending inhibition of spinal pain transmission. Exp Neurol 261: 475-485, 2014.

9. Du J, Yang X, Zhang L and Zeng YM: Expression of TRPM8 in the distal cerebrospinal fluid-contacting neurons in the brain mesencephalon of rats. Cerebrospinal Fluid Res 6: 3, 2009.

10. Lu X, Geng X, Zhang L, Zeng Y, Dong H and Yu H: Substance $\mathrm{P}$ expression in the distal cerebrospinal fluid-contacting neurons and spinal trigeminal nucleus in formalin-induced the orofacial inflammatory pain in rats. Brain Res Bull 78: 139-144, 2009.

11. Miyajima M, Nakajima M, Motoi Y, Moriya M, Sugano H, Ogino I, Nakamura E, Tada N, Kunichika M and Arai H: Leucine-rich $\alpha 2$-glycoprotein is a novel biomarker of neurodegenerative disease in human cerebrospinal fluid and causes neurodegeneration in mouse cerebral cortex. PLoS One 8: e74453, 2013

12. Sainaghi PP, Collimedaglia L, Alciato F, Molinari R, Sola D, Ranza E, Naldi P, Monaco F, Leone M, Pirisi M and Avanzi GC: Growth arrest specific gene 6 protein concentration in cerebrospinal fluid correlates with relapse severity in multiple sclerosis. Mediators Inflamm 2013: 406483, 2013.

13. Wang MS and Zhang LC: Methodological comparison on the tracing CSF-CNs with HRP and CB-HRP. Acad Med 12: 286-287, 1992 .

14. Lu X, Geng X, Zhang L and Zeng Y: The methodology for labeling the distal cerebrospinal fluid-contacting neurons in rats. J Neurosci Methods 168: 98-103, 2008.

15. Zhang MM, Ji W, Pei LY, Wang W, Chen T, Wang W, Li H, Zhang T, Wu SX and Li YQ: Acute colitis induces neurokinin 1 receptor internalization in the rat lumbosacral spinal cord. PLoS One 8: e59234, 2013.

16. Palecek J, Paleckova V and Willis WD: Postsynaptic dorsal column neurons express NK1 receptors following colon inflammation. Neuroscience 116: 565-572, 2003.

17. Palecek J, Paleckova V and Willis WD: Fos expression in spinothalamic and postsynaptic dorsal column neurons following noxious visceral and cutaneous stimuli. Pain 104: 249-257, 2003.

18. Miampamba M, Chéry-Croze S, Gorry F, Berger F and Chayvialle JA: Inflammation of the colonic wall induced by formalin as a model of acute visceral pain. Pain 57: 327-334, 1994.

19. Bai L, Wang W, Dong YL, Wang W, Huang J, Wang XY, Wang LY, Li YQ and Wu SX: Attenuation of mouse somatic and emotional inflammatory pain by hydralazine through scavenging acrolein and inhibiting neuronal activation. Pain Physician 15: 311-326, 2012.

20. Calle M, Claassen IE, Veening JG, Kozicz T, Roubos EW and Barendregt HP: Opioid peptides, CRF, and urocortin in cerebrospinal fluidcontacting neurons in Xenopus laevis. Ann N Y Acad Sci 1040: 249-252, 2005.

21. Vigh B, Manzano e Silva MJ, Frank CL, Vincze C, Czirok SJ, Szabó A, Lukáts A and Szél A: The system of cerebrospinal fluid-contacting neurons. Its supposed role in the nonsynaptic signal transmission of the brain. Histol Histopathol 19: 607-628, 2004.

22. Wu TT, Zhao ZJ, Xu C and Zhang LC: Distribution of TRPC6 in the cerebrospinal fluid-contacting nucleus of rat brain parenchyma and its expression in morphine dependence and withdrawal. Neurochem Res 36: 2316-2321, 2011.

23. Wang CG, Ding YL, Zheng TF, Wei JQ, Liu H, Chen YF, Wang JY and Zhang LC: Extracellular signal-regulated kinase 5 in the cerebrospinal fluid-contacting nucleus contributes to morphine physical dependence in rats. J Mol Neurosci 50: 215-220, 2013.

24. Laird JM, Olivar T, Roza C, De Felipe C, Hunt SP and Cervero F: Deficits in visceral pain and hyperalgesia of mice with a disruption of the tachykinin NK1 receptor gene. Neuroscience 98: 345-352, 2000

25. Palecek J: The role of dorsal columns pathway in visceral pain. Physiol Res 53 (Suppl 1): S125-S130, 2004. 